\title{
A Comparative Analysis of Microscopy, Coproantigen Serology, and Nested Multiplex PCR in the Laboratory Diagnosis of Entamoeba histolytica Infection
}

\author{
Sindhusuta Das ${ }^{1}$ Nonika Rajkumari ${ }^{1}$ Anitha Gunalan ${ }^{1}$ Dhanalakshmi Rajavelu ${ }^{1}$ Jeby Jose Olickal ${ }^{2}$
}

${ }^{1}$ Department of Microbiology, Jawaharlal Institute of Postgraduate Medical Education \& Research, Puducherry, India

2 Department of Preventive and Social Medicine, Jawaharlal Institute of Postgraduate Medical Education \& Research, Puducherry, India

J Lab Physicians 2022;14:125-131.
Address for correspondence Nonika Rajkumari, MBBS, MD, Department of Microbiology, Jawaharlal Institute of Postgraduate Medical Education \& Research, Puducherry, 605006, India (e-mail: nonika.raj@gmail.com).

\section{Abstract \\ Keywords \\ - amoebiasis \\ - coproantigen testing \\ - Entamoeba histolytica \\ - amoebic colitis \\ - amoebic dysentery}

Objectives Amoebiasis is caused by the most common intestinal protozoan parasite Entamoeba histolytica. This parasite causes amoebic colitis, which is manifested by diarrhea, followed by dysentery. The laboratory diagnosis of intestinal amoebiasis in most cases is by microscopic examination of stool samples. Other nonroutine methods include coproantigen enzyme-linked immunosorbent assay (ELISA) from stool samples, serum ELISA for antibodies, stool culture, isoenzyme analysis, and polymerase chain reaction (PCR). The present study aimed to comparatively analyze the different diagnostic modalities used for the detection of $E$. histolytica from the stool sample of patients with intestinal amoebiasis.

Materials and Methods This study was undertaken with 631 patients, during a period of 3 years, from January 2017 to December 2019. Stool specimen obtained from each patient was subjected to direct microscopic wet mount examination, coproantigen ELISA, and nested multiplex PCR, respectively.

Results Out of all the patients tested, 5.2\% were positive for E. histolytica. Among the positive cases, stool microscopy was positive in $3.17 \%$, coproantigen ELISA was positive in $29(4.6 \%)$ cases, and PCR was positive in 30 (4.75\%) cases.

Statistical Analysis The prevalence of $E$. histolytica infection was summarized as percentages. The three diagnostic tests done were statistically analyzed, taking microscopy as the gold standard. The agreement between techniques (microscopy, coproantigen ELISA, and PCR) was analyzed with kappa statistics. Sensitivity, specificity, positive predictive value, negative predictive value, and diagnostic accuracy were summarized as percentage with $95 \%$ confidence interval.

Conclusion In all suspected amoebiasis cases, a combination of stool microscopy, coproantigen testing with molecular detection of the parasite offers the best approach to diagnosis of this parasitic infection. published online July 22, 2021
DOI https://doi.org/

$10.1055 / \mathrm{s}-0041-1732488$ ISSN 0974-2727.
(C) 2021. The Indian Association of Laboratory Physicians. All rights reserved.

This is an open access article published by Thieme under the terms of the Creative Commons Attribution-NonDerivative-NonCommercial-License, permitting copying and reproduction so long as the original work is given appropriate credit. Contents may not be used for commercial purposes, or adapted, remixed, transformed or built upon. (https://creativecommons.org/ licenses/by-nc-nd/4.0/)

Thieme Medical and Scientific Publishers Pvt. Ltd., A-12, 2nd Floor, Sector 2, Noida-201301 UP, India 


\section{Introduction}

Amoebiasis is caused by the intestinal protozoan parasite Entamoeba histolytica. Owing to the lack of adequate sanitation and increased fecal contamination of water supplies, this parasitic disease is more commonly seen in developing countries. ${ }^{1}$ Apart from cases of $E$. histolytica infection, there have been other case reports of E. dispar and E. moshkovskii being isolated from patients with gastrointestinal symptoms, but there is no convincing evidence of a causal association between the isolation of these two species and the clinical manifestations in the cases reported. ${ }^{2}$ Nowadays, it is also observed that Entamoeba moshkovskii is emerging as another protozoan parasite causing infantile diarrhea. ${ }^{3,4}$

The fourth leading parasitic cause of mortality worldwide is attributed to amoebiasis and data reveal that it caused 11,300 deaths globally in the year $2013 .{ }^{5}$ Many people get infected with $E$. histolytica, making amoebic colitis a leading cause of diarrhea, which kills more than 55,000 people each year, owing to its severe complications. ${ }^{6}$ In India, approximately 15 to $20 \%$ of the population is affected by this protozoan parasite. ${ }^{7}$ The clinical features of amoebiasis range from asymptomatic colonization to extraintestinal invasive amoebiasis. Usually, the affected cases are asymptomatic, but invasive intestinal infection also manifests in certain patients, which has a range of clinical features like chronic abdominal cramps, tenesmus, watery diarrhea or dysentery, and loss of weight. ${ }^{8} \mathrm{~A}$ prompt diagnosis is crucial for the patients with amoebic dysentery and asymptomatic cases so as to interrupt the transmission of disease. The laboratory diagnosis of E. histolytica infection is challenging, and the currently available diagnostic methods lack sensitivity. In the developing countries, intestinal amoebiasis is usually diagnosed in laboratory by identifying parasite cysts or motile trophozoites by wet mount examination of the suspected stool samples. The drawbacks of this conventional method include its low sensitivity and specificity, with false positive results common, owing to the presence of other protozoans like E. dispar or E. moshkovskii. Ideally, diagnosis should be framed taking into consideration a combination of laboratory testing methods, namely the microscopic detection of parasite in stool specimens, serodiagnosis by detection of $E$. histolytica-specific coproantigen, and stool polymerase chain reaction (PCR) for detection of parasite DNA.

The present study aimed to comparatively analyze the currently used different diagnostic modalities for the detection of E. histolytica from stool sample of patients clinically diagnosed with intestinal amoebiasis.

\section{Materials and Methods}

This cross-sectional study was conducted from January 2017 to December 2019 in a tertiary care hospital, Puducherry, south India. Ethical approval for the study was obtained from the Jawaharlal Institute of Postgraduate Medical Education \& Research (JIPMER) Institute ethics committee. Considering a prevalence of $E$. histolytica as $4.5 \%$ with 1.35 absolute precision, the calculate sample size for the study was 734 . We have included all eligible patients visited outpatient department (OPD) during the study period.

A group of 631 participants included in the study and their results were analyzed. All the subjects who reported to the hospital OPD with complaints of abdominal pain, vomiting, diarrhea, dysentery, indigestion, flatulence, dehydration, and weight loss were included. The cases with suspected bacterial causes of gastroenteritis and noninfectious etiology were excluded from the study. About 5 to $8 \mathrm{~g}$ of stool sample was collected in a $25 \mathrm{~mL}$, sterile, screw-capped container, on 3 consecutive days from each patient. A written informed consent was obtained from all the study participants. A detailed history of prior treatment with any antiamoebic drugs or antibiotics was taken at the time of collection of stool samples from the clinically suspected patients. The unpreserved stool samples were transported to the laboratory for processing within 1 hour of collection. After reaching the laboratory, macroscopic examination of stool sample for noting the color, consistency, presence of blood, mucus, etc. was done. Then, each stool sample was divided into three parts. The first part was used for direct wet mount examination and formol ether sedimentation concentration method, according to Garcia et al. ${ }^{9}$ The second and third part of stool sample were stored at $-20^{\circ} \mathrm{C}$ for coproantigen detection by enzyme-linked immunosorbent assay (ELISA) and PCR, respectively.

\section{Direct Stool Microscopy}

Fresh unpreserved stool samples were subjected to a wet mount examination in $0.9 \%$ saline and Lugol's iodine. Each wet mount was microscopically examined for pus cells, mucus flecks, red blood cells and for the presence of E. histolytica quadrinucleate cysts and trophozoites. The trophozoites of a typical $E$. histolytica are approximately 20 to $30 \mu \mathrm{m}$ in diameter. It has a nucleus containing a central endosome, peripheral chromatin deposition, and radial achromatic fibrils, which gives the appearance of "cart-wheel" morphology. The serial stepwise motility of $E$. histolytica trophozoite from a patient sample is demonstrated in - Fig. 1A. Mostly, the mature parasite cysts are spherical, quadrinucleate, and measure approximately 10 to $15 \mu \mathrm{m}$ in diameter ( $\mathbf{- F i g . ~ 1 B )}$. The stool samples were also concentrated by formol-ether sedimentation technique, by the procedure given by Garcia et al, ${ }^{9}$ where wet mount was inconclusive or negative. Apart from a
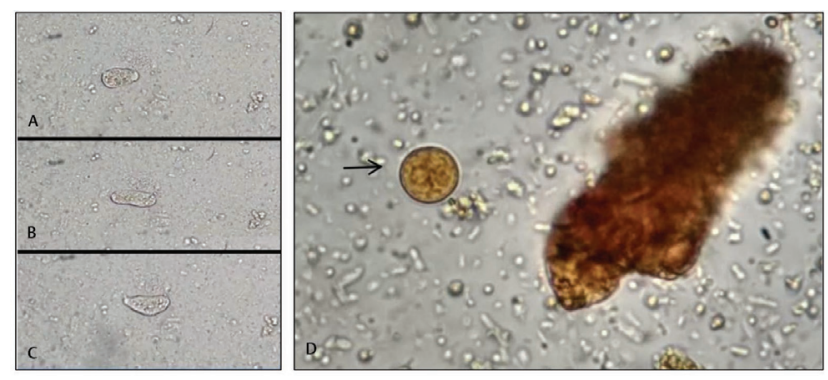

Fig. 1 (A,B,C) The serial stepwise locomotion images of a motile Entamoeba histolytica trophozoite found in a positive stool sample of a case. (D) The quadrinucleate cyst of E. histolytica found in the stool sample of a positive case. 
standard wet mount, the stool specimens were subjected to a modified iron-hematoxylin staining.

\section{Coproantigen Detection by ELISA}

All the second part of patient's stool samples was batched and E. histolytica ELISA was performed once a week. Each stool sample was subjected to coproantigen detection ELISA according to the manufacturer's instructions (Savyon Diagnostics Ltd, Israel). For detection of antigen in each stool sample, $100 \mu \mathrm{L}$ of diluted stool specimen was added to one of the antibody-coated microtiters well of the plate. It was incubated for 1 hour at $37^{\circ} \mathrm{C}$ and washed. Then, $100 \mu \mathrm{L}$ of horseradish peroxidase-conjugate was added and incubated for 1 hour at $37^{\circ} \mathrm{C}$ and washed. After that, $100 \mu \mathrm{L}$ of 3,3',5,5'tetramethylbenzidine-substrate was added, incubated for 15 minutes at room temperature. The reaction was terminated by addition of $100 \mu \mathrm{L}$ of stop solution. Finally, the absorbance was read at $450 \mathrm{~nm}$ using an ELISA reader. According to the manufacturer's instructions, a test was considered positive when the optical density reading of a sample was $>0.15$ at $450 \mathrm{~nm}$. The kit had a sensitivity of $100 \%$ and specificity of $95.4 \%$.

\section{Nested Multiplex PCR}

The third part of each stool sample was subjected to nested multiplex PCR targeting the 16S-like rRNA gene for simultaneous detection and differentiation of E. histolytica, E. moshkovskii, and E. dispar, according to the method given by Khairnar and Parija. ${ }^{10}$ The parasite nucleic acid extraction from the stool specimens was performed by a cetyltrimethylammonium bromide extraction method. The extracted DNA was then quantified in a spin column and purified DNA extract from stool specimens was determined by ultraviolet (UV) absorbance using a double beam spectrophotometer. The genus-specific primers sequences used for the first PCR were E-1 5' TAAGATGCACGAGAGCGAAA 3' (forward primer) E-2 5' GTACAAAGGGCAGGGACGTA 3' (reverse primer). The species-specific primer sequences used in the second nested PCR were E. histolytica species EH-1 5' AAGCATTGTTTCTAGATCTGAG 3' (forward primer) EH-2 5' AAGAGGTCTAACCGAAATTAG 3' (reverse primer), E. moshkovskii species Mos-15' GAAACCAAGAGTTTCACAAC 3' (forward primer) Mos-2 5' CAATATAAGGCTTGGATGAT 3' (reverse primer), and E. dispar species ED-1 5' TCTAATTTCGATTAGAACTCT 3' (forward primer) ED-2 5' TCCCTACCTATTAGACATAGC 3' (reverse primer), respectively. For a reaction volume of $25 \mu \mathrm{L}$, comprising $2.5 \mu \mathrm{L}$ of 10XPCR buffer (Biogene), $1.5 \mu \mathrm{L}$ of $25 \mathrm{mM} \mathrm{MgCl}_{2}$ (Bangalore Genei Ltd), $1.4 \mu \mathrm{L}$ of deoxynucleoside triphosphate mix ( $5 \mathrm{mM}$ each dNTP, ABgene), $0.3 \mu \mathrm{L}(5 \mathrm{IU} / \mu \mathrm{L})$ of Taq polymerase (Biogene Ltd), $0.3 \mu \mathrm{M}$ of each primer (IDT) and $2.5 \mu \mathrm{L}$ of template DNA was added in genus-specific and species-specific PCR. The PCR reaction tubes were then placed in a thermal cycler. The PCR mix underwent an initial denaturation at $96^{\circ} \mathrm{C}$ for 2 minutes, followed by 30 cycles of PCR. Each amplification cycle consisted of $92^{\circ} \mathrm{C}$ for 60 seconds (denaturation), $56^{\circ} \mathrm{C}$ for 60 seconds (annealing), and $72^{\circ} \mathrm{C}$ for 90 seconds (extension). Finally, one cycle of extension at $72^{\circ} \mathrm{C}$ for 7 minutes was performed. In the species-specific nested multiplex PCR (which had multiple primer sets in the same tube), only the annealing temperature was modified. About $3 \mu \mathrm{L}$ of the amplification products were subjected to electrophoresis through $1.8 \%$ agarose gel at $120 \mathrm{~V}$ for 45 -minute duration, and were visualized by ethidium bromide dye staining under UV light, for bands of target DNA detected. The PCR product giving bright band at 439 bp was considered positive for E. histolytica, whereas all other bands were taken as negative for E. histolytica (- Figs. 2A, B). Among the other bands, the PCR product giving bright bands at 553 and $174 \mathrm{bp}$ was considered positive for $E$. moshkovskii and E. dispar, respectively. Positive and negative control reactions were included with each batch of samples analyzed by nested multiplex PCR. The positive control used for Entamoeba and the individual species were an inhouse designed positive controls, obtained after sequencing the highly conserved region. A negative sample, which was proven PCR negative, was used as the negative control. The nested multiplex PCR detected E. histolytica, E. dispar, and E. moshkovskii DNA at a rate of 1,000 parasites/0.05 $g$ of feces. The detection limit of nested multiplex PCR for E. histolytica was 25 Entamoeba protozoa cells.

\section{Statistical Analysis}

The study data for analysis were entered into Microsoft Excel sheet. The three diagnostic tests done were statistically analyzed, taking microscopy as the gold standard. The agreement between techniques (microscopy, coproantigen ELISA and PCR) was analyzed with kappa statistics. Sensitivity, specificity, positive predictive value (PPV), negative predictive value (NPV), and diagnostic accuracy were summarized as percentage with $95 \%$ confidence interval. All the statistical analysis was performed with IBM SPSS Statistics 20 and Open Epiversion 3.01 software.

\section{Results}

A total of 631 patients participated in the study. The unpreserved stool samples from all the 631 patients were subjected to three different diagnostic modalities for diagnosis of Entamoeba spp. infections. The three tests used were stool microscopy, coproantigen ELISA, and nested multiplex PCR. A positive result obtained in any one of the above three diagnostic tests used was considered as a positive case.

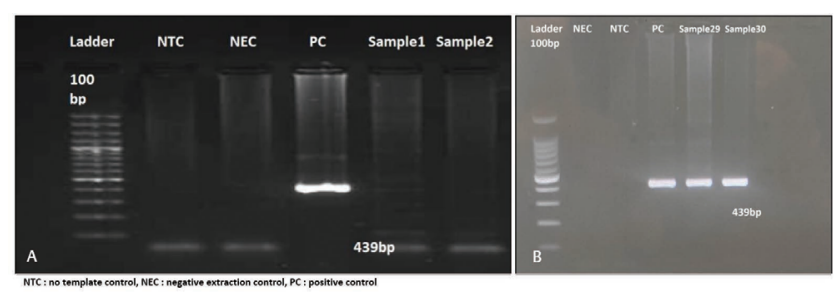

Fig. 2 Amplified products of PCR were analyzed by agarose gel electrophoresis. The size of the amplification product is indicated on the right (in base pairs). Samples 1 and 2 are negative for Entamoeba histolytica and the PCR products showing the bright band at 439 bp (samples 29 and 30 ) are positive for $E$. histolytica, along with the positive and negative controls used for the nested multiplex PCR. NTC, no template control; NEC, negative extraction control; PC, positive control; PCR, polymerase chain reaction. 
Among the 631 patients, 33 (5.2\%) patients were positive for E. histolytica infection. The multiplex PCR results revealed 30 (4.7\%) cases to be positive for E. histolytica, 9 (1.4\%) cases to be positive for Entamoeba dispar, and $3(0.4 \%)$ cases to be positive for E. moshkovskii cysts in stool sample of patients. These two species, that is, E. dispar and E. moshkovskii, were not included for analysis.

Among the Entamoeba histolytica infection cases, analysis of the different diagnostic techniques used showed that, stool microscopy was positive in 20 (3.17\%) cases and negative in 611 (96.83\%) cases, coproantigen ELISA was positive in $29(4.6 \%)$ cases and negative in 602 (95.4\%) cases, and multiplex PCR was positive in 30 (4.75\%) cases and negative in 601 (95.25\%) cases (-Table 1). It was also observed that the most commonly affected age group was between 26 and 45 years and the disease was more prevalent among the male gender (-Table 2). The individual E. histolytica positive cases were tabulated according to the results obtained by the diagnostic method results used (-Table 3 ). Further, the percentage shows the \% positivity among the three stool samples collected from each positive case (-Table $\mathbf{3}$ ).

The statistical analysis was done, taking microscopy as the gold standard. The statistical agreement between microscopy and coproantigen ELISA was found to be 0.79, using kappa statistics with 95\% confidence interval (-Table 4). The validity indicators of coproantigen ELISA against microscopy showed a sensitivity of $90 \%$, specificity of $98.2 \%$, PPV of $62.07 \%$, NPV of $99.67 \%$, and a diagnostic accuracy of $97.94 \%$ (-Table 5). The statistical agreement between microscopy and PCR was found to be 0.72 , using kappa statistics with $95 \%$ confidence interval (- Table 6). The validity indicators of PCR against microscopy showed a sensitivity of $100 \%$, specificity of $98.36 \%$, PPV of $66.67 \%$, NPV of $100 \%$, and a diagnostic accuracy of $98.42 \%$ (- Table 7 ).

\section{Discussion}

The present study detected $5.2 \%$ cases to be positive for E. histolytica among a total of 631 patients tested over a duration of 3 years. This study showed male gender was more commonly affected, belonging to the age group 26 to 45 years. Similar findings were also noted by Tharmaratnam et al and Kantor et al in their study. 4,11

From the very old days, the laboratory diagnosis of E. histolytica has always been based on direct microscopic examination of stool samples showing distinct protozoan morphology. ${ }^{12}$ Currently, however, usage of the direct microscopy-based identification techniques to differentiate among protozoa with similar morphological features like E. coli, E. moshkovskii, E. dispar, and E. hartmanni is seemingly unreliable. ${ }^{2}$ There is a vast diversity in the morphological appearances of the cysts and trophozoites of Entamoeba species. Therefore, the confirmed identification of these intestinal parasites requires observation by a skilled microscopist. Moreover, various studies prove that the sensitivity and specificity of conventional microscopy, on a single stool specimen, for distinguishing the various species of Entamoe$b a$ are far less than reliable. ${ }^{13,14}$ In fresh wet mount preparation, the locomotion of E. histolytica in fresh preparations usually occurs linearly, with the clear hyaline ectoplasm flowing to form blunt-ended pseudopodia, then leading the granular endoplasm containing the nucleus to flow in the same direction. ${ }^{15}$ At times, when a fresh stool specimen cannot be examined immediately, it may be preserved with a fixative like polyvinyl alcohol or maintained at $4^{\circ} \mathrm{C}$ temperature. Stool specimens can be examined either unstained or stained with Lugol's iodine. Iodine increases the contrast and improves the clarity of internal structures like nucleus of the parasite. Wheatley's trichrome staining or modified iron hematoxylin stains for permanent smears have been suggested as better stains for regular use in the diagnosis of E. histolytica or E. dispar. ${ }^{16-18}$ There are several factors that adversely affect the results of microscopy. These include lack of trained microscopists; delayed transport of sample to the laboratory; difficulty in differentiation between nonmotile trophozoites and polymorphonuclear leukocytes or macrophages; inappropriate sample collection; interfering substances such as antibiotics, purgatives, antacids, cathartics, antidiarrheal preparations (kaolin or bismuth), or enemas; inadequate number of specimens collected (at least three specimens are needed due to intermittent shedding nature of the parasite cyst); lack of preservation of stool specimens with fixatives; and presence of other commensal amoebae. ${ }^{19}$ CDC guidelines say that multiple stool samples (at least 3) should be tested before a negative result is reported, and stool samples in formalin, or other fixatives, need to be concentrated prior to microscopic examination ${ }^{20}$ and finally the choice of diagnostic techniques should depend on available equipment and reagents, experience, and considerations of time and cost.

In the present study, it was observed that coproantigen ELISA was positive in 29 (4.6\%) cases and negative in 602 (95.4\%) cases. The validity indicators of coproantigen ELISA against microscopy showed a sensitivity of $90 \%$, specificity of $98.2 \%$, PPV of $62.07 \%$, NPV of $99.67 \%$, and a diagnostic accuracy of $97.94 \%$. This is similar to the study findings by el-Hamshary et al and Bayoumy et al, where they have concluded that coproantigen assay using ELISA is more sensitive and specific than microscopy even when the parasitic count is low, thus reducing the chances of missing

Table 1 Result showing the comparative performance of different diagnostic methods ( $n=631)$

\begin{tabular}{|l|l|l|l|l|l|l|}
\hline Result & Microscopy & $\%$ & Serology by coproantigen ELISA & $\%$ & PCR & $\%$ \\
\hline Positive & 20 & 3.17 & 29 & 4.60 & 30 & 4.75 \\
\hline Negative & 611 & 96.83 & 602 & 95.40 & 601 & 95.25 \\
\hline
\end{tabular}

Abbreviations: ELISA, enzyme-linked immunosorbent assay; PCR, polymerase chain reaction. 
Microscopy, Coproantigen Serology, and Nested Multiplex PCR in Entamoeba histolytica Infection Das et al. 129

Table 2 Age and sex distribution of the positive cases $(n=33)$

\begin{tabular}{|l|l|l|l|l|}
\hline Age & Male & $\%$ & Female & $\%$ \\
\hline $0-14$ & 1 & 3.03 & 0 & 0 \\
\hline $15-25$ & 3 & 9.09 & 1 & 3.03 \\
\hline $26-35$ & 5 & 15.2 & 4 & 12.2 \\
\hline $36-45$ & 8 & 24.2 & 2 & 6.06 \\
\hline $46-55$ & 1 & 3.03 & 3 & 9.09 \\
\hline$>55$ & 5 & 15.2 & 0 & 0 \\
\hline Total & 23 & 69.7 & 10 & 30.3 \\
\hline
\end{tabular}

Table 3 Matrix showing the result of testing method of each positive cases with \% positivity among the 3 stool samples of each case $(n=33)$

\begin{tabular}{|l|l|l|l|}
\hline SI. no. & Microscopy & ELISA & PCR \\
\hline 1 & $+(100 \%)$ & $+(100 \%)$ & $+(100 \%)$ \\
\hline 2 & $+(100 \%)$ & $+(100 \%)$ & $+(100 \%)$ \\
\hline 3 & $+(100 \%)$ & $+(100 \%)$ & $+(100 \%)$ \\
\hline 4 & $+(66.6 \%)$ & - & $+(66.6 \%)$ \\
\hline 5 & $+(66.6 \%)$ & $+(66.6 \%)$ & $+(66.6 \%)$ \\
\hline 6 & - & $+(66.6 \%)$ & $+(66.6 \%)$ \\
\hline 7 & - & $+(66.6 \%)$ & - \\
\hline 8 & - & - & $+(66.6 \%)$ \\
\hline 9 & $+(100 \%)$ & $+(100 \%)$ & $+(100 \%)$ \\
\hline 10 & $+(100 \%)$ & $+(100 \%)$ & $+(100 \%)$ \\
\hline 11 & $+(100 \%)$ & $+(100 \%)$ & $+(100 \%)$ \\
\hline 12 & $+(66.6 \%)$ & - & $+(66.6 \%)$ \\
\hline 13 & $+(100 \%)$ & $+(100 \%)$ & $+(100 \%)$ \\
\hline 14 & - & $+(66.6 \%)$ & - \\
\hline 15 & - & $+(66.6 \%)$ & $+(66.6 \%)$ \\
\hline 16 & - & - & $+(66.6 \%)$ \\
\hline 17 & $+(100 \%)$ & $+(100 \%)$ & $+(100 \%)$ \\
\hline 18 & - & $+(66.6 \%)$ & - \\
\hline 19 & $+(100 \%)$ & $+(100 \%)$ & $+(100 \%)$ \\
\hline 20 & $+(100 \%)$ & $+(100 \%)$ & $+(100 \%)$ \\
\hline 21 & $+(100 \%)$ & $+(100 \%)$ & $+(100 \%)$ \\
\hline 22 & $+(100 \%)$ & $+(100 \%)$ & $+(100 \%)$ \\
\hline 23 & $+(100 \%)$ & $+(100 \%)$ & $+(100 \%)$ \\
\hline 24 & $+(100 \%)$ & $+(100 \%)$ & $+(100 \%)$ \\
\hline 25 & $+(100 \%)$ & $+(100 \%)$ & $+(100 \%)$ \\
\hline 26 & - & $+(66.6 \%)$ & $+(100 \%)$ \\
\hline 27 & - & $+(66.6 \%)$ & $+(100 \%)$ \\
\hline 28 & - & $+(100 \%)$ & $+(100 \%)$ \\
\hline 29 & $+(100 \%)$ & $+(100 \%)$ & $+(100 \%)$ \\
\hline 30 & $+(100 \%)$ & $+(100 \%)$ & $+(100 \%)$ \\
\hline 31 & - & $+(66.6 \%)$ & $+(66.6 \%)$ \\
\hline 32 & $+(100 \%)$ & $+(100 \%)$ \\
\hline 33 & $+(100 \%)$ & $+(100 \%)$ \\
\hline
\end{tabular}

Abbreviations: ELISA, enzyme-linked immunosorbent assay; PCR, polymerase chain reaction.
Table 4 Agreement between gold standard (microscopy) and ELISA

\begin{tabular}{|l|l|l|l|}
\hline \multirow{2}{*}{ ELISA } & \multicolumn{2}{|l|}{ Microscopy } & \multirow{2}{*}{$\begin{array}{l}\text { Kappa } \\
(95 \% \mathrm{CI})\end{array}$} \\
\cline { 2 - 3 } & Positive & Negative & \\
\hline Positive & 18 & 11 & \multirow{2}{*}{$0.79(0.72-0.87)$} \\
\hline Negative & 2 & 600 & \\
\hline Total & 20 & 611 & \\
\hline
\end{tabular}

Abbreviations: $\mathrm{Cl}$, confidence interval; ELISA, enzyme-linked immunosorbent assay.

Table 5 Validity indicators of ELISA against microscopy

\begin{tabular}{|l|l|}
\hline Parameter & Estimate $(95 \% \mathrm{CI})$ \\
\hline Sensitivity & 90 \\
\hline Specificity & 98.2 \\
\hline PPV & 62.07 \\
\hline NPV & 99.67 \\
\hline Likelihood ratio positive test & 49.99 \\
\hline Likelihood ratio negative test & 0.1018 \\
\hline Diagnostic accuracy & 97.94 \\
\hline
\end{tabular}

Abbreviations: $\mathrm{Cl}$, confidence interval; NPV, negative predictive value; PPV, positive predictive value.

Table 6 Agreement between gold standard (microscopy) and PCR

\begin{tabular}{|l|l|l|l|}
\hline \multirow{2}{*}{ PCR } & \multicolumn{2}{|l|}{ Microscopy } & \multirow{2}{*}{$\begin{array}{l}\text { Kappa } \\
\text { (95\% CI) }\end{array}$} \\
\cline { 2 - 3 } & Positive & Negative & \\
\hline Positive & 20 & 10 & \multirow{2}{*}{$0.72(0.65-0.80)$} \\
\hline Negative & 0 & 601 & \\
\hline Total & 20 & 611 & \\
\hline
\end{tabular}

Abbreviations: $\mathrm{Cl}$, confidence interval; $\mathrm{PCR}$, polymerase chain reaction.

Table 7 Validity indicators of PCR against microscopy

\begin{tabular}{|l|l|}
\hline Parameter & Estimate $(95 \% \mathrm{Cl})$ \\
\hline Sensitivity & 100 \\
\hline Specificity & 98.36 \\
\hline PPV & 66.67 \\
\hline NPV & 100 \\
\hline Likelihood ratio positive test & 61.1 \\
\hline Likelihood ratio negative test & 0.0 \\
\hline Diagnostic accuracy & 98.42 \\
\hline
\end{tabular}

Abbreviations: $\mathrm{Cl}$, confidence interval; NPV, negative predictive value; $\mathrm{PCR}$, polymerase chain reaction; PPV, positive predictive value.

positive cases even in the asymptomatic cases and widespread use of this technique has allowed for revising the epidemiology of the true pathogenic E. histolytica, thus eliminating the need for unnecessary treatment. ${ }^{21,22}$ These tests may be helpful from a diagnostic perspective in developed nations, where infections due to E. histolytica are not very commonly seen. The coproantigen detection ELISA is 
more useful as an adjunct to microscopic diagnosis in detecting intestinal parasites, than antibody detection ELISA. Recent studies reveal regarding the high sensitivity and specificity of fecal antigen assay with the use of polyclonal or monoclonal antibodies that can successfully detect Entamoeba infections, which are negative by stool microscopy. ${ }^{23,24}$ These antigenbased detection tests have a sensitivity approaching that of stool culture. ${ }^{2}$ A good sensitivity and specificity have been observed for tests detecting of E. histolytica antigen in stool specimens, especially in patients with amoebic colitis and asymptomatic cases. ${ }^{25}$ Importantly, among the routinely used diagnostic methods, that is, antigen detection, antibody detection, microscopy, and isoenzyme analysis, PCR., only coproantigen detection using ELISA is technically simple to perform and can be used in resource poor settings that do not have molecular facilities, thus making it appropriate for use in the developing world, where amoebiasis is more commonly seen. ${ }^{26}$ The only drawback of this coproantigen testing is that it can be performed only on unpreserved fresh or frozen stool samples, since fecal antigens get destroyed by fixative agents. ${ }^{27}$ Also, sensitivity of coproantigen tests is lower than molecular methods of detection. ${ }^{28}$

Due to the many limitations of the conventional techniques, molecular methods of detection are emerging as a handy tool in the diagnosis of various infections including amoebiasis. The accurate identification of pathogenic $E$. histolytica is essential in the treatment of the affected patients as well as studying the epidemiology of amoebiasis outbreaks. The World Health Organization also recommends the application of PCR technology for the accurate detection and differentiation of $E$. histolytica from other commensal amoebae, directly from stool samples. ${ }^{2}$ Blessmann et al developed a closed tube real-time PCR to detect $E$. histolytica directly from stool samples. They concluded in their study that the two sets of primers are actually species specific and that PCR is not influenced by the presence of considerable amounts of other Entamoeba species. ${ }^{29}$ In the current study, the nested multiplex PCR technique was used for the detection of E. histolytica directly from stool sample, by the method given by Khairnar and Parija. ${ }^{10}$ This nested multiplex PCR detected E. histolytica, E. dispar, and E. moshkovskii DNA, even at the minimum parasite concentration tested $(1,000$ parasites $/ 0.05 \mathrm{~g}$ of feces). The detection limit of this PCR for $E$. histolytica, E. dispar, and E. moshkovskii was approximately 25 Entamoeba protozoa cells. In the present study, it was observed that the nested multiplex PCR was positive in 30 (4.75\%) cases and negative in 601 (95.25\%) cases for $E$. histolytica. The possible causes of PCR negative samples among the serology positive cases might be attributable to very low parasite density in stool or an extraintestinal infection. The validity indicators of PCR against microscopy showed a sensitivity of $100 \%$, specificity of $98.36 \%$, PPV of $66.67 \%$, NPV of $100 \%$, and a diagnostic accuracy of $98.42 \%$. This is similar to the study findings by Blessmann et al. ${ }^{29}$ In comparison to microscopy or coproserology, PCR detected slightly higher number of positive samples, suggesting that PCR is more sensitive than either of those techniques. The study by Weitzel et al study suggests that PCR testing would be a great aid in the laboratory diagnosis of amoebiasis, which will surpass the limitations of conventional modes of diagnosis of this parasitic disease. ${ }^{30}$ In endemic setups, which usually the developing countries are, it is advisable to perform all the three tests simultaneously, so as to diagnose all the cases and treat accordingly. But with respect to cost-effectiveness, microscopy and serology can be performed in all suspected cases and PCR can be done only for cases with strong clinical suspicion despite a negative microscopy and serology result.

\section{Conclusion}

This study throws substantial light on the diagnostic advantages of PCR over the conventional coproantigen ELISA-based kits and stool microscopy, in both sensitivity and specificity. In addition, the nested multiplex PCR has the advantage of specifically targeting and detecting $E$. histolytica, E. dispar, and E. moshkovskii in clinical stool samples. This study aimed to discuss different methods that exist for the laboratory identification of E. histolytica. After statistical analysis of the observations from the present study, it is thus concluded that in all cases of clinically suspected amoebiasis, preferably a combination of stool microscopy, stool coproantigen testing followed up by molecular detection of the parasite DNA from stool samples offers the best and robust approach to laboratory diagnosis of E. histolytica.

\section{Informed Consent}

Written informed consent was taken from all adult participants and parents of minor participants, who agreed to be enrolled in the study group, after getting approval from the JIPMER Ethical Committee, JIPMER.

\section{Authors' Contribution}

Sindhusuta Das was responsible for sample and data collection, and its processing including microscopy, coproELISA, and PCR. She was responsible for writing the manuscript and its submission. Nonika Rajkumari was responsible for the conceptualization of the study, counterchecking the results, and monitoring of the work performed and analysis of the results. She also checked and refined the manuscript and gave critical inputs to the paper. Anitha Gunalan helped in data collection and initial data processing. Dhanalakshmi Rajavelu helped in data collection and the figure refinement. Jeby Jose Olickal helped in the statistical modeling of the study selection of the study parameters as well as gave critical inputs to the study and helped in refining the final manuscript.

\section{Conflict of Interest}

The authors declare no conflicts of interest.

\section{References}

1 Zulfiqar H, George M, Shawn H“Amebiasis.” In StatPearls Treasure Island (FL): StatPearls Publishing, 2020 http://www.ncbi.nlm. nih.gov/books/NBK519535/ Accessed June 11, 2021 
2 Fotedar R, Stark D, Beebe N, Marriott D, Ellis J, Harkness J. Laboratory diagnostic techniques for Entamoeba species. Clin Microbiol Rev 2007;20(03):511-532

3 Singh PPInfectious Diseases and Your Health. First edition. Singapore: Springer2018

4 Kantor M, Abrantes A, Estevez Aet al. Entamoeba histolytica: updates in clinical manifestation, pathogenesis, and vaccine development. Can J Gastroenterol Hepatol 2018;2018:4601420

5 Wang H, Naghavi M, Allen C, et al. GBD 2015 Mortality and Causes of Death Collaborators. Global, regional, and national life expectancy, all-cause mortality, and cause-specific mortality for 249 causes of death, 1980-2015: a systematic analysis for the Global Burden of Disease Study 2015. Lancet 2016;388(10053)1459-1544

6 Shirley DT, Farr L, Watanabe K, Moonah S. A review of the global burden, new diagnostics, and current therapeutics for amebiasis. Open Forum Infect Dis 2018;5(07):ofy161

7 Shimokawa C, Kabir M, Taniuchi Met al.Entamoeba moshkovskii is associated with diarrhea in infants and causes diarrhea and colitis in mice. J Infect Dis 2012;206(05):744-751

8 Parija SC, Mandal J, Ponnambath DK. Laboratory methods of identification of Entamoeba histolytica and its differentiation from look-alike Entamoeba spp. Trop Parasitol 2014;4(02):90-95

9 Garcia LS, Arrowood M, Kokoskin Eet al.Practical guidance for clinical microbiology laboratories: laboratory diagnosis of parasites from the gastrointestinal tract. Clin Microbiol Rev 2017; 31(01):e00025-17 Doi: 10.1128/CMR.00025-17

10 Khairnar K, Parija SC. A novel nested multiplex polymerase chain reaction (PCR) assay for differential detection of Entamoeba histolytica, E. moshkovskii and E. dispar DNA in stool samples. BMC Microbiol 2007;7:47. Doi: 10.1186/1471-2180-7-47

11 Tharmaratnam T, Kumanan T, Iskandar MAet al.Entamoeba histolytica and amoebic liver abscess in northern Sri Lanka: a public health problem. Trop Med Health 2020;48(01):2 Doi: 10.1186/ s41182-020-0193-2

12 Tanyuksel M, Petri WA Jr. Laboratory diagnosis of amebiasis. Clin Microbiol Rev 2003;16(04):713-729

13 Garcia LS, Bruckner DADiagnostic Medical Parasitology. 3rd edition. Washington, D.C.: ASM Press; 1997

14 Markell EK, John DT, Krotoski WALumen-Dwelling Protozoa. 8th edition. Philadelphia: The W. B. Saunders Co.; 1999

15 Proctor EM. Laboratory diagnosis of amebiasis. Clin Lab Med 1991;11(04):829-859PubMedWeb of ScienceGoogle Scholar

16 Palmer J. Modified iron hematoxylin/kinyoun stain. Clin Microbiol Newsl 1991;13:39-40(Letter.) [Google Scholar]
17 Scholten TH, Yang J. Evaluation of unpreserved and preserved stools for the detection and identification of intestinal parasites. Am J Clin Pathol 1974;62(04):563-567[PubMed] [Google Scholar]

18 Yang J, Scholten T. A fixative for intestinal parasites permitting the use of concentration and permanent staining procedures. Am J Clin Pathol 1977;67(03):300-304[PubMed] [Google Scholar]

19 “CDC - DPDx - Diagnostic Procedures - Stool Specimens," January 9, 2019. https://www.cdc.gov/dpdx/diagnosticprocedures/ stool/specimencoll.html. Accessed June 12, 2021

20 "CDC-DPDx - Amebiasis - Laboratory Diagnosis." Accessed July 16, 2020. https://www.cdc.gov/dpdx/amebiasis/dx.html

21 el-Hamshary EM, el-Shewy KA, Hegazy MM, Zakaria H. Diagnostic potentials of copro-antigen detection based ELISA, compared to microscopy in intestinal amoebiasis. J Egypt Soc Parasitol 2004;34 (02):601-610

22 BayousA,TahaM,TarekZ,HeshamG,WallE. Coproantigen versus classical microscopy as a diagnostic tool for entamoeba histolytica infection in the egyptian patients. Egypt J Hosp Med 2019; 74(6):1424-1427

23 Urdaneta H, Guimarães S, Silva EF, Tavares CA. Entamoeba histolytica: detection of coproantigens by purified antibody in the capture sandwich ELISA. Rev Inst Med Trop São Paulo 1994; 36(06):539-545

24 Kabil SM, Hassan MM, Atta M. el Ghysha O. ELISA in detection of Entamoeba histolytica antigens in stools. J Egypt Soc Parasitol 1990;20(02):673-676

25 Petri WA Jr, Singh U. Diagnosis and management of amebiasis. Clin Infect Dis 1999;29(05):1117-1125

26 Haque R, Ali IKM, Akther S. Petri WA Jr. Comparison of PCR, isoenzyme analysis, and antigen detection for diagnosis of Entamoeba histolytica infection. J Clin Microbiol 1998;36(02): 449-452

27 Saidin S, Othman N, Noordin R. Update on laboratory diagnosis of amoebiasis. Eur J Clin Microbiol Infect Dis 2019;38(01):15-38

28 Gonin P, Trudel L. Detection and differentiation of Entamoeba histolytica and Entamoeba dispar isolates in clinical samples by PCR and enzyme-linked immunosorbent assay. J Clin Microbiol 2003;41(01):237-241

29 Blessmann J, Heidrun B, Phuong Aet al.Real-time PCR for detection and differentiation of Entamoeba histolytica and Entamoeba dispar in fecal samples. J Clin Microbiol 2002;40(12):4413-4417

30 Weitzel T, Cabrera J, Rosas Ret al.Enteric multiplex PCR panels: a new diagnostic tool for amoebic liver abscess? New Microbes New Infect 2017;18:50-53 